\title{
Améliorer la prévision de température en montagne par des descentes d'échelle
}

\author{
Gabriel Arnould', Ingrid Dombrowski-Etchevers'1, \\ Isabelle Gouttevin'2, Yann Seity'
}

1. CNRM, Université de Toulouse / Météo-France / CNRS, Toulouse

2. Université Grenoble Alpes, Université de Toulouse / Météo-France / CNRS / CNRM / Centre d'études de la neige, Grenoble

gabriel.arnould@meteo.fr

\section{Résumé}

En montagne, la prévision du temps et d'aléas aussi dangereux et localisés que les avalanches requiert une modélisation de l'atmosphère à haute résolution spatiale. Palliant des simulations coûteuses, des méthodes de descente d'échelle ont été appliquées pour produire des champs météorologiques à $500 \mathrm{~m}$ de résolution, sur les massifs alpins, à partir des prévisions issues du modèle Arome à $1,3 \mathrm{~km}$. L'évaluation s'est concentrée sur la température à 2 mètres, paramètre météorologique clé pour la physique du manteau neigeux. Une descente d'échelle exploite un profil thermique environnemental. Sa pertinence est démontrée dans certaines configurations météorologiques, mais les erreurs intrinsèques au modèle Arome contraignent son potentiel.
Dans l'atmosphère, la température diminue avec l'altitude : en moyenne, le gradient vertical de température au sein de la troposphère est de $-6,5^{\circ} \mathrm{C} / \mathrm{km}$. Cette moyenne est calculée sur un ensemble de profils atmosphériques observés en tous points du globe. Cependant, le profil de température en un point donné dépend des conditions atmosphériques qui lui sont associées. Dans un environnement sec, le gradient vertical approche la valeur de $-9,8{ }^{\circ} \mathrm{C} / \mathrm{km}$. $\mathrm{Au}$ contraire, il peut être supérieur à $-6,5{ }^{\circ} \mathrm{C} / \mathrm{km}$ au sein d'un environnement saturé où la libération de chaleur latente par condensation freine la
Ces phénomènes ont une forte incidence sur les activités humaines. La figure 1 illustre les plus courants.

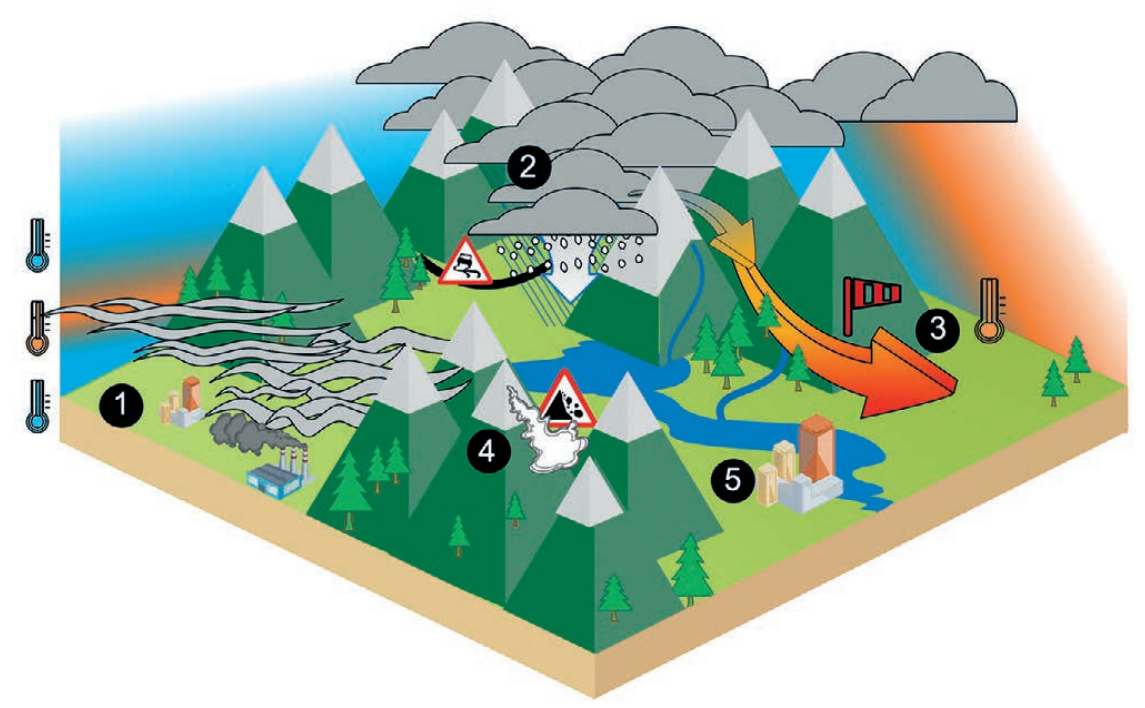

Figure 1. Quelques phénomènes météorologiques et aléas communs à la montagne : (1) inversion thermique ; (2) neige par isothermie ; (3) fœehn ; (4) risque d'avalanches ; (5) risque de crues liées à la fonte nivale. 


\section{Abstract}

\section{Downscaling to improve air temperature forecast in mountain areas}

In mountain areas, a numerical model with high spatial resolution is necessary to forecast the local meteorological conditions and the natural hazard such as avalanches. To limit time-consuming computations, downscaling methods were used to produce meteorological fields within a grid spacing of $500 \mathrm{~m}$ in the Alps from the Météo-France $1.3 \mathrm{~km}$ resolution forecast model (Arome). The evaluation focuses on the 2 meters temperature which is a key variable in snowpack changes. One method employs a correction based on a local vertical temperature lapse rate. Its impact is quite positive in some atmospheric conditions but remains limited by the Arome bias. décroissance de la température avec l'altitude. Par ailleurs, lors des nuits calmes et dégagées, le bilan radiatif en surface est négatif : le sol perd plus d'énergie qu'il n'en reçoit. Ainsi la température diminue-t-elle à son voisinage, à tel point que l'air peut y être plus froid qu'en altitude. Une inversion thermique apparaît alors et son évolution dépend grandement de la topographie du milieu. Le sommet de la couche d'inversion, souvent matérialisé par des stratus, empêche le renouvellement d'air et piège les particules polluantes (Paci et al., 2016).

La transformation de l'eau de l'état liquide à l'état gazeux, ou de l'état solide à l'état liquide, est endothermique : le changement d'état prélève de la chaleur dans l'environnement et diminue ainsi sa température. À la saison froide, quand une perturbation se bloque sur les reliefs, des précipitations continues se produisent. La fonte des flocons de neige et l'évaporation des gouttes de pluie sont donc susceptibles de refroidir à $0{ }^{\circ} \mathrm{C}$ une colonne d'atmosphère où la température était initialement positive. Dans cette couche isotherme $\left(\right.$ à $\left.0{ }^{\circ} \mathrm{C}\right)$, la limite pluie-neige s'abaisse naturellement, ce qui peut surprendre le trafic routier au fond des vallées.

Lorsqu'un flux rencontre un relief, l'air se soulève, se refroidit et la vapeur d'eau qu'il contient se condense, puis forme des précipitations. Après le passage du relief, l'air asséché descend le versant sous le vent. Son réchauffement tend alors vers la valeur de $9,8{ }^{\circ} \mathrm{C} / \mathrm{km}$. Un épisode de fœhn est ainsi propice à une fonte rapide $d u$ manteau neigeux qui peut être à l'origine de crues.

Ces spécificités constituent un véritable défi pour la prévision numérique du temps. La capacité d'un modèle à simuler de tels phénomènes dépend généralement de sa résolution en cohérence avec la finesse de son orographie. Celle-ci est de $1,3 \mathrm{~km}$ pour le modèle de prévision numérique du temps Arome. C'est adapté à la représentation des processus de mésoéchelle, comme la convection par exemple. Cependant, en terrain complexe, c'est encore trop lâche pour prévoir la température (à $2 \mathrm{~m}$ ), le vent (à $10 \mathrm{~m}$ ) ou l'humidité (à $2 \mathrm{~m}$ ) à l'échelle très locale, d'autant plus que l'échelle résolue (pour laquelle les phénomènes sont bien décrits par le modèle) est toujours plus grande que la résolution spatiale du modèle.
Pourtant, les variations de ces paramètres influencent fortement l'évolution du manteau neigeux dont la modélisation dépend des prévisions atmosphériques. À terme, leur imprécision peut donc altérer l'anticipation du risque d'avalanche ou l'appréhension des crues. Ces enjeux ont motivé la mise en place de stratégies qui visent à produire des prévisions à plus haute résolution en zone de montagne sur la base des champs bruts d'Arome $1,3 \mathrm{~km}$ : on parle de « descente d'échelle ».

\section{Deux approches de descente d'échelle}

Cette étude a eu pour objectif de fournir des champs météorologiques sur une grille à 500 mètres de résolution, soit une précision plus que doublée par rapport à la version opérationnelle du modèle Arome. Pour cela, deux approches ont été abordées.

Une descente d'échelle dynamique consiste à faire tourner le modèle initial à plus haute résolution spatiale. $\mathrm{La}$ finesse de l'orographie est par conséquent accentuée. Arome-Recherche $500 \mathrm{~m}$, en cours de développement au Centre national de recherche météorologique (CNRM), a été utilisé. Sur son domaine de calcul, les conditions aux limites sont fournies par Arome $1,3 \mathrm{~km}$, version opérationnelle. La résolution verticale est identique à celle d'Arome 1,3 km qui dispose de 90 niveaux dont 23 sont situés dans les 1000 premiers mètres au-dessus de la surface, le premier étant à 5 mètres. Cette méthode présente l'avantage d'utiliser toute la dynamique et la physique du modèle. Son inconvénient tient à son temps de calcul élevé qui limite son utilisation à des fins opérationnelles.

Une descente d'échelle corrective peut être une solution à ce problème. Cette fois-ci, on raisonne directement sur les prévisions brutes du modèle Arome $1,3 \mathrm{~km}$ en leur appliquant des corrections adaptées afin de tenir compte de la variabilité topographique de petite échelle. Dans cette étude, plusieurs méthodes de descente d'échelle corrective ont été appliquées. Le travail s'est concentré sur la température à 2 mètres $(T 2 m)$, paramètre météorologique clé pour l'évolution du manteau neigeux. 


\section{Méthodes de descente d'échelle corrective pour la température}

Lors du passage de la grille grossière d'un modèle à la résolution cible, une descente d'échelle corrective considère trois étapes dont les traitements sont schématisés sur la figure 2 pour la température à 2 mètres.

1. La variabilité horizontale du paramètre étudié peut simplement être traduite par un procédé d'interpolation qui cherche à construire un champ continu à partir de données discrètes, à savoir les sorties du modèle sur sa grille grossière. Pour cela, il faut estimer la fonction mathématique qui donne l'évolution du paramètre entre les points du modèle. Si on considère une relation de proportionnalité, on aura recours à une interpolation dite bilinéaire. Cette approche est souvent privilégiée, car elle permet d'assurer une certaine continuité dans le champ calculé. Par conséquent, elle induit un effet de lissage qui s'avère irréaliste lorsque la température montre des gradients naturels importants, particulièrement en montagne. En comparaison, on peut aussi associer à chaque point de la grille cible la valeur du point le plus proche sur la grille du modèle. Dans ce cas, on échappe au lissage des champs, mais une partie de l'information spatiale est perdue. Cette interpolation au point voisin est néanmoins pertinente si la maille initiale n'est pas trop lâche, ce qui est le cas du modèle Arome 1,3 km. 2. En montagne, l'évolution spatiale d'un paramètre météorologique est soumise à des gradients à la fois horizontaux et verticaux. Ces derniers résultent surtout des variations du relief auxquelles la température est très sensible. Ainsi une correction de hauteur est-elle souhaitable pour tenir compte de la différence d'altitude entre les points du modèle et les points cibles lors de l'interpolation horizontale. Cette correction est fondée sur le gradient vertical de température noté $\Gamma$, si tant est qu'on connaisse sa valeur! 3. De nombreux effets ont encore été occultés. Il y a par exemple le rayonnement et le chauffage différentiel : si le point cible appartient à un versant orienté vers le nord (ubac) et si l'interpolation ne considère que des points situés sur des versants orientés vers le sud (adret), la correction d'altitude ne permettra pas de traduire l'écart résultant en température. De plus, à cause de la résolution, la signature de certains phénomènes très localisés n'apparaît pas dans les sorties du modèle (brises, turbulence, inversions dans des vallées étroites...). Selon les conditions atmosphériques et les propriétés du milieu, des traitements spécifiques peuvent chercher à les retranscrire dans une descente d'échelle.

\section{Quelle valeur pour le gradient vertical de température $\Gamma$ ?}

Une réponse simple serait de prendre $\Gamma$ égal à sa valeur moyenne observée à la surface du globe, soit $-6,5^{\circ} \mathrm{C} / \mathrm{km}$. Or, comme nous l'avons vu en introduction, l'évolution de la température à 2 mètres avec l'altitude du relief n'est pas uniforme dans le temps et dans l'espace (Soci et al., 2011). Elle dépend de l'humidité, de la stratification des masses d'air qui, au voisinage du sol, est fortement liée aux échanges d'énergie entre la surface et l'atmosphère.
Une méthode plus sophistiquée, développée par Sheridan et al. (2010), consiste à calculer un gradient « local » pour chaque point de la grille fine. La figure 3 illustre le procédé. Par souci de clarté, un seul point cible (en jaune) est représenté. La grille de surface du modèle est matérialisée par des points rouges (et un point bleu, le plus proche du point cible). On considère un certain nombre de points modèle (ici 16) situés dans l'environnement du point cible. En reportant leurs données de température $(T)$ et d'altitude $(z)$ sur le profil de droite (étoiles rouges ou bleues), on peut déduire une fonction $T(z)$ qui approxime la valeur de $T$ pour une altitude donnée : on parle de régression. En faisant l'hypothèse d'une dépendance linéaire entre $T$ et $z$, cette fonction est une droite (trait pointillé noir) et la valeur de sa pente nous donne le gradient thermique vertical associé à l'environnement du point cible. Il suffit ensuite de prendre la température $T_{i}$ au point le plus proche (en bleu) et

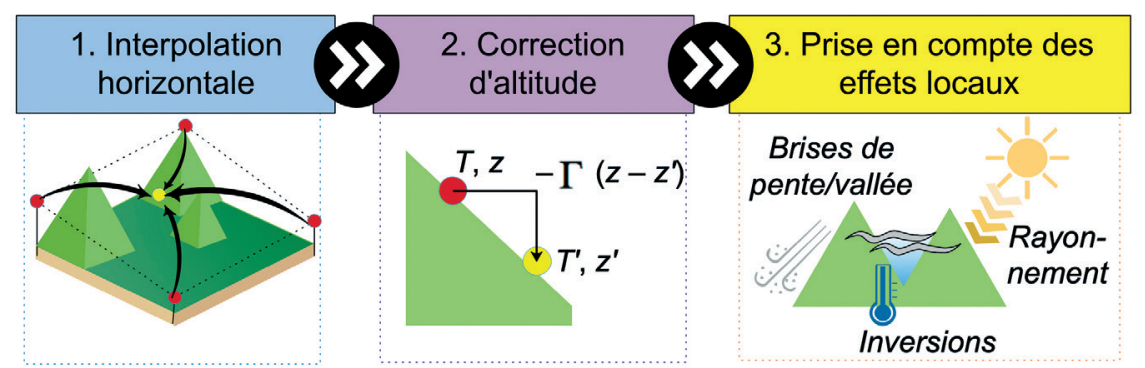

Figure 2. Les trois étapes de traitement impliquées dans une descente d'échelle corrective de la température à 2 mètres.

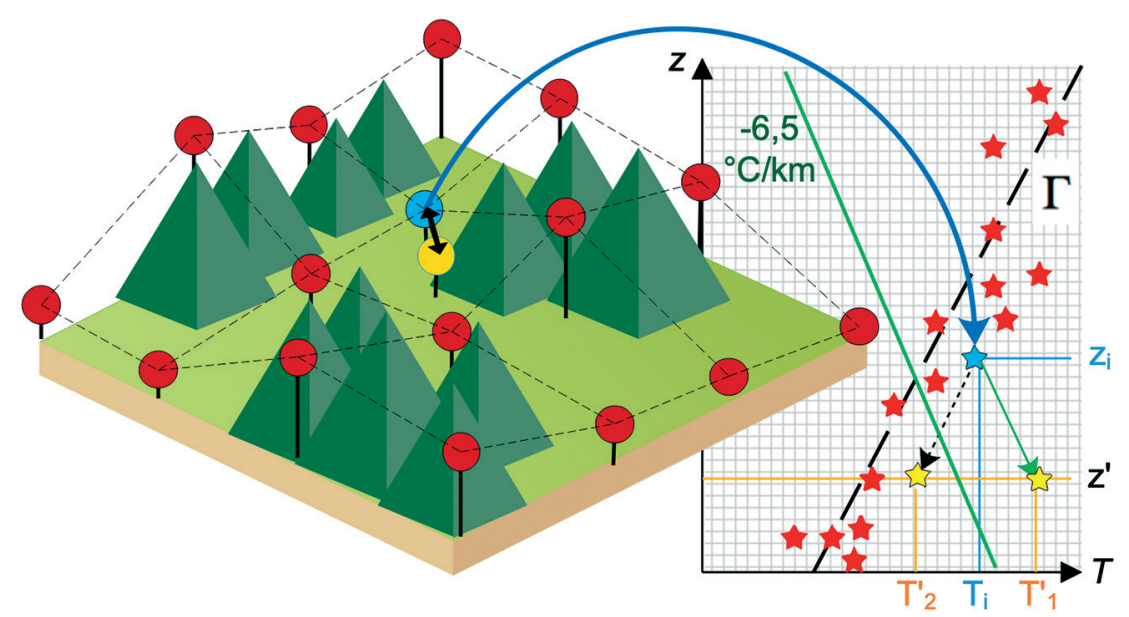

Figure 3. Illustration de la méthode de Sheridan et al. (2010). Le relief représenté correspond à la topographie réelle. Le point cible figure en jaune. Les points du modèle sont en rouge (ou en bleu pour le voisin du point cible). Le maillage évoqué par les tirets noirs donne donc un aperçu du relief implémenté dans le modèle. Comme on peut le remarquer, le point cible est logiquement plus proche du relief réel que les autres points. On déduit de la régression linéaire entre ces données $T(z)$ un gradient vertical local $\Gamma$. Ici $\Gamma$ est positif et représente donc un profil d'inversion. II est ainsi très éloigné du gradient " standard " à $-6,5^{\circ} \mathrm{C} / \mathrm{km}$ (trait vert) dont le choix surestimerait largement la température du point cible $\left(T_{1}^{\prime}>T_{2}^{\prime}\right)$. 
de lui soustraire ce gradient multiplié par la différence d'altitude avec le point cible. La température de celui-ci est alors $T_{2}^{\prime}=T_{i}-\left(z-z^{\prime}\right)$. Cette méthode présuppose que le modèle est capable de simuler des gradients thermiques pertinents, même si son orographie grossière nuit au réalisme des champs finaux de température à 2 mètres.

\section{Descentes d'échelle pour la température et stratégies d'évaluation}

Une descente d'échelle dynamique (Arome-Recherche $500 \mathrm{~m}$ ) et trois descentes d'échelle correctives ont été évaluées pour la $T 2 m$ :

- Une interpolation horizontale bilinéaire (inter.bilin.) + une correction d'altitude avec $\Gamma=-6,5{ }^{\circ} \mathrm{C} / \mathrm{km}$. En réalité, cette approche cache trois sousétapes : (1) on calcule les températures du modèle sur un niveau d'altitude de référence via l'application du gradient $\Gamma$, (2) on réalise l'interpolation bilinéaire sur ce niveau de référence, (3) on ramène la température interpolée au niveau réel de la grille fine via l'application du gradient $\Gamma$. De cette façon, on assure l'indépendance de la correction horizontale (sous-étape 2) par rapport à la correction verticale (sous-étapes 1 et 3). L'approche décrite ici est déjà utilisée à l'opérationnel pour traiter les sorties brutes du modèle. Ces dernières, alors écrites sur une grille de calcul à la géométrie non régulière, sont projetées sur une grille latitude/longitude préférée pour le travail des prévisionnistes.

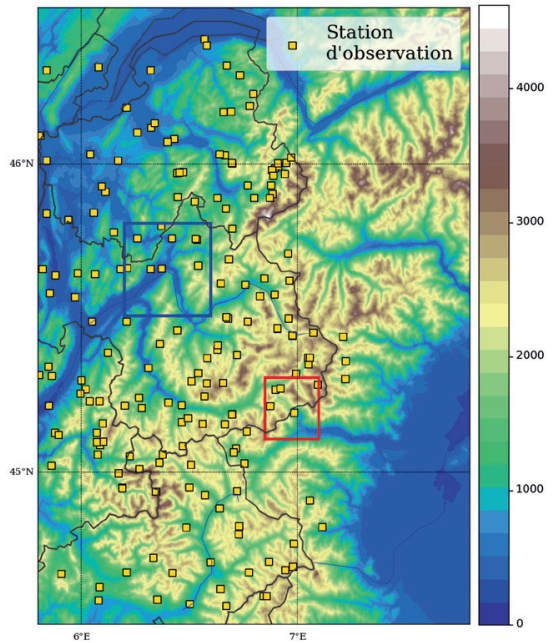

Figure 4. Relief du domaine d'étude (en m) vu par la grille à $500 \mathrm{~m}$ de résolution. La première étude de cas se focalise sur la zone délimitée par le cadre rouge, la seconde par le cadre bleu.

\section{Influence du protocole de comparaison prévision/observation sur le calcul des scores}

Pour le calcul des scores de $T 2 m$, l'Organisation météorologique mondiale (OMM) recommande de ramener la valeur prévue $T^{\prime}$ à l'altitude $z_{\text {obs }}$ de la station (température observée notée $T_{\text {obs }}$ ) afin d'éliminer les écarts liés à la différence d'altitude. Elle préconise l'application du gradient standard de $-6,5^{\circ} \mathrm{C} / \mathrm{km}$ dont le profil associé figure en vert sur le graphique ci-dessous. Certes, il s'agit de la valeur moyenne observée sur la planète. II n'en demeure pas moins éloigné de la réalité dans de nombreuses situations, surtout en montagne (Soci et al., 2011). Le cas le plus flagrant est celui des inversions thermiques où la décroissance de la température avec l'altitude lui est opposée. Sur la figure, la température $T^{\prime}$ est issue d'une descente d'échelle reposant sur un gradient local appliqué à la température $T$ du modèle grossier. Dans cet exemple, on a supposé que le modèle grossier était parfait. Le profil local, figuré par le trait pointillé rouge, l'est donc également, si bien que les points

$T_{\text {oss }}\left(z_{\text {obs }}\right), T(z)$ et $T^{\prime}\left(z^{\prime}\right)$ sont alignés sur la figure. Or, en ramenant $T^{\prime}$ au niveau zobs via la correction classique, une différence nette apparaît entre la température $T^{\prime}$ obtenue et $T_{\text {obs }}$, alors qu'elle devrait être nulle. En l'absence de correction, ce faux biais persiste entre T' et Tobs, mais il est de moindre amplitude. Une alternative consisterait à appliquer une correction fondée sur le gradient local. On aurait ainsi $T_{\text {cor }}^{\prime}=T_{\text {obs }}$, ce qui est encore mieux. Cette alternative n'a toutefois pas été retenue, car elle ne serait pas cohérente avec les descentes d'échelle fondées sur un gradient standard, ni avec la descente d'échelle dynamique Arome-Recherche 500 m. Pour limiter une dégradation artificielle des scores tout en assurant une certaine robustesse dans le protocole de comparaison prévision/ observation, il a donc été décidé d'exclure les stations pour lesquelles la différence d'altitude avec la grille cible est trop importante (Vionnet et al., 2016) et de n'appliquer aucune correction pour les autres.

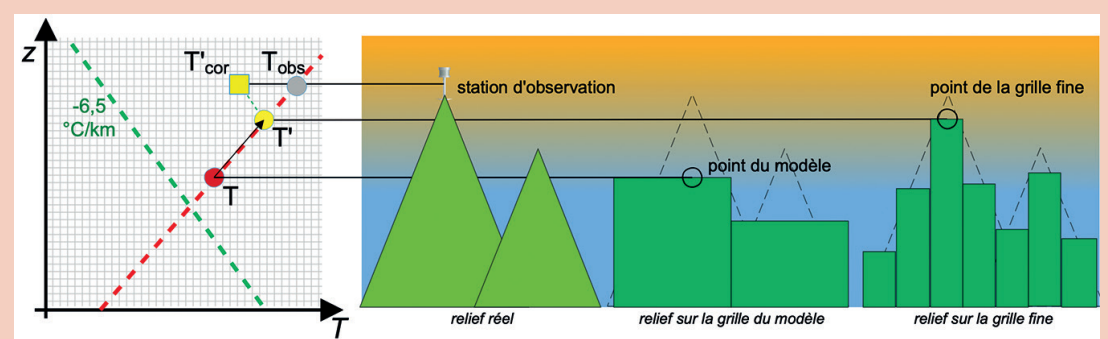

Dégradation des scores résultant de l'application du gradient standard à une prévision parfaite de température en situation d'inversion.

Un tel changement de grille suggère une étape d'interpolation et de correction verticale.

- Une interpolation horizontale au point le plus proche (voisin) + une correction d'altitude avec $\Gamma=-6,5{ }^{\circ} \mathrm{C} /$ $\mathrm{km}$. Cette interpolation ne lisse pas le champ de température, contrairement à l'interpolation bilinéaire, et peut préserver la variabilité spatiale d'Arome à $1,3 \mathrm{~km}$.

- Une interpolation horizontale au point le plus proche (voisin) + une correction d'altitude avec un gradient local calculé via la méthode de Sheridan et al. (2010). Une interpolation bilinéaire ne serait pas adaptée pour cette méthode, car le gradient local est calculé pour les points de la grille fine uniquement. On ne pourrait donc pas réaliser la sous-étape (1) mentionnée dans la première approche.

Dans un premier temps, ces simulations ont été évaluées sur la période hivernale 2019-2020 (novembre 2019 à mars 2020). Les descentes d'échelle correctives ont été réalisées à partir de la prévision quotidienne du modèle
Arome $1,3 \mathrm{~km}$ (réseau de $0 \mathrm{~h} \mathrm{TU}$ ) utilisée sur les 24 premières heures d'échéance. Une descente d'échelle dynamique étant supposée plus performante qu'une approche purement corrective, la prévision quotidienne du modèle de recherche Arome $500 \mathrm{~m}$ sert a priori de référence. Les scores sont calculés par rapport à des observations in situ, ces dernières étant réparties sur le domaine d'étude qui comprend une large portion des Alpes françaises et italiennes (figure 4). Lorsqu'on compare la $T 2 m$ observée et la $T 2 m$ prévue au point de grille le plus proche, il serait tentant d'introduire une correction qui tient compte de la différence d'altitude éventuelle entre le site d'observation et ce point de grille. Or, comme cela est expliqué dans l'encadré 1 , cette pratique est ici écartée afin d'assurer une certaine cohérence avec les différentes méthodes de descente d'échelle et afin de ne pas dégrader les scores associés. Par conséquent n'ont été gardées que les stations dont la différence d'altitude avec le point de grille le plus proche est inférieure à 150 mètres - protocole déjà utilisé par Vionnet et al. (2016) -, 
portant tout de même à 182 le nombre de sites sélectionnés sur le domaine. Dans un second temps, l'étude s'est concentrée sur deux situations types reportées dans les comptes rendus mensuels des prévisionnistes de Météo-France pour la région Auvergne-Rhône-Alpes (communication personnelle de Joël Marceaux). Cette analyse introduit quelques éléments de discussion.

\section{Résultats sur les Alpes françaises}

Le biais moyen ( $T 2 m$ prévue - T2m observée), calculé sur la période et le domaine d'étude en fonction de l'heure TU et de la simulation, est visible en figure 5. Afin d'évaluer l'effet de l'altitude sur le comportement de l'erreur, trois tranches ont été discriminées: 300-1000 m (43 stations), 1000 $1800 \mathrm{~m}$ (66 stations), $1800 \mathrm{~m}$ et plus (73 stations). La ligne noire correspondrait à une simulation parfaite (biais nul). Les descentes d'échelle correctives ont toutes des scores similaires. Quelques divergences sont à noter pour la tranche d'altitude la plus basse où les approches reposant sur une interpolation horizontale au point le plus proche (Voisin $+\Gamma=-6,5^{\circ} \mathrm{C} / \mathrm{km}$ et Voisin $+\Gamma$ local) sont moins éloignées des observations que la simulation réalisée avec une interpolation bilinéaire (Inter. bilin. $\left.+\Gamma=-6,5^{\circ} \mathrm{C} / \mathrm{km}\right)$. De plus, sous $1000 \mathrm{~m}$, la méthode de Sheridan et al. (Voisin $+\Gamma$ local) est la plus performante, notamment aux heures froides (nuit et matin) où un gradient local est le plus à même de retranscrire un profil d'inversion. L'erreur associée à la descente d'échelle dynamique est plus faible à basse altitude, mais contre toute attente, se retrouve accentuée dès la tranche intermédiaire : au-dessus de $1800 \mathrm{~m}$, son biais froid est compris entre $3,5^{\circ} \mathrm{C}$ (à $0 \mathrm{~h}$ TU) et $1,5^{\circ} \mathrm{C}$ (à $15 \mathrm{~h} \mathrm{TU}$ ) quand les méthodes correctives affichent un biais froid situé entre $3{ }^{\circ} \mathrm{C}$ et $1{ }^{\circ} \mathrm{C}$. En parallèle, l'écart type a été calculé pour chaque simulation. Ce dernier indique la dispersion de l'erreur autour de sa moyenne (le biais). Il n'est ici pas négligeable, avec des valeurs de 2 à $3{ }^{\circ} \mathrm{C}$ selon l'altitude pour toutes les simulations. Une opération de débiaisage, qui consisterait à retrancher systématiquement aux champs calculés la valeur de leur erreur moyenne, est donc peu pertinente.

\section{Deux situations météorologiquement intéressantes}

Le comportement des descentes d'échelle correctives est ici analysé sur des situations concrètes : un épisode de fœhn et une inversion thermique matinale. En dépit des biais constatés dans la section précédente, notamment en altitude, le modèle Arome $500 \mathrm{~m}$ est encore considéré comme référence, l'idée étant de critiquer la variabilité spatiale des champs obtenus plutôt que des valeurs ponctuelles uniquement.

\section{Un épisode de fohn}

Le 13 février 2020, un front chaud aborde les Alpes par l'ouest. Côté français, la perturbation apporte des précipitations dont la persistance fait baisser la limite pluie/neige par isothermie : il neigera jusqu'à $500 \mathrm{~m}$ d'altitude dans certaines vallées, une situation non prévue par le modèle Arome 1,3 km. La descente du flux perturbé côté italien se traduit par un épisode de fœhn classique dans les Alpes cottiennes. La figure 6 représente le champ de $T 2 m$ issu d'Arome $500 \mathrm{~m}$ et de deux descentes d'échelle correctives (Inter. bilin. $+\Gamma=-6,5^{\circ} \mathrm{C} / \mathrm{km}$ et Voisin $+\Gamma$ local) au niveau du val de Suse (cadre rouge sur la figure 4). Les sorties du modèle Arome opérationnel à 1,3 km de résolution apparaissent également. On remarque que l'imprécision de son orographie entraîne une sous-estimation du réchauffement de la masse d'air dans la branche nord de la vallée, mais une surestimation en altitude, au niveau de la station de Barcenisio. Concernant l'allure du champ, les
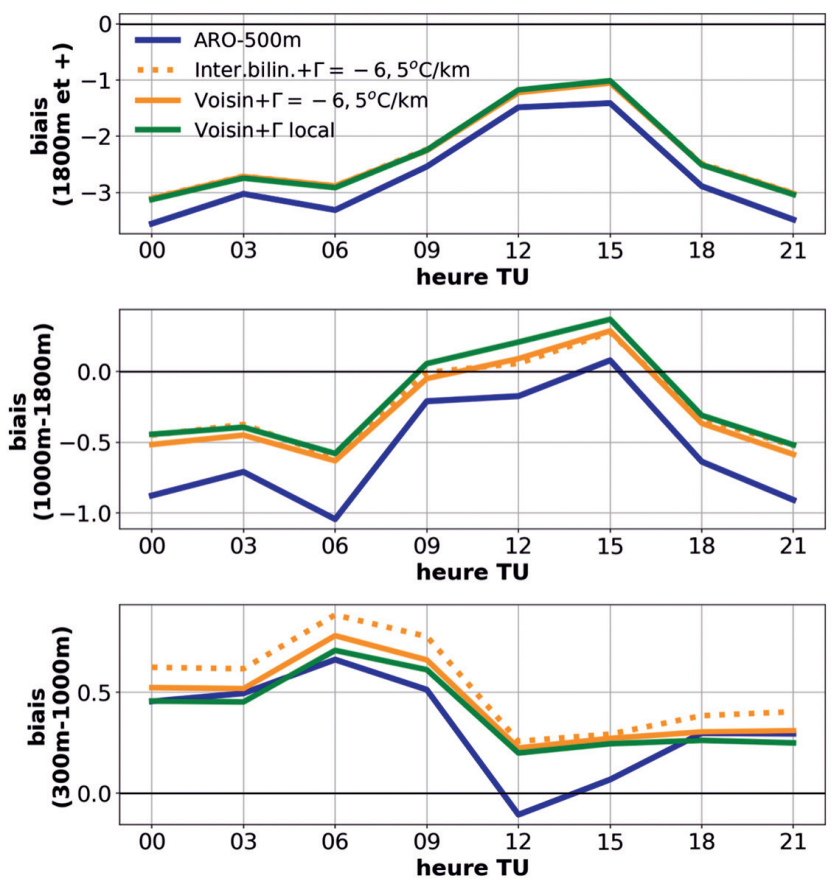

Figure 5. Évolution diurne du biais de $T 2 m$ (en ${ }^{\circ} \mathrm{C}$ ) calculé pour chaque simulation et pour les trois niveaux d'altitude discriminés.

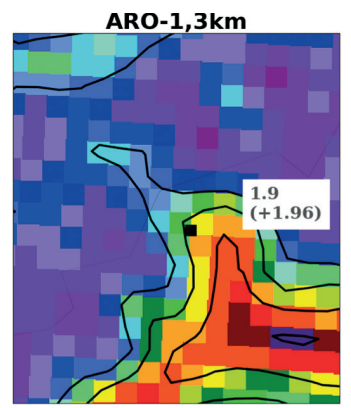

Inter.bilin. $+\Gamma=-6,5^{\circ} \mathbf{C} / \mathbf{k m}$
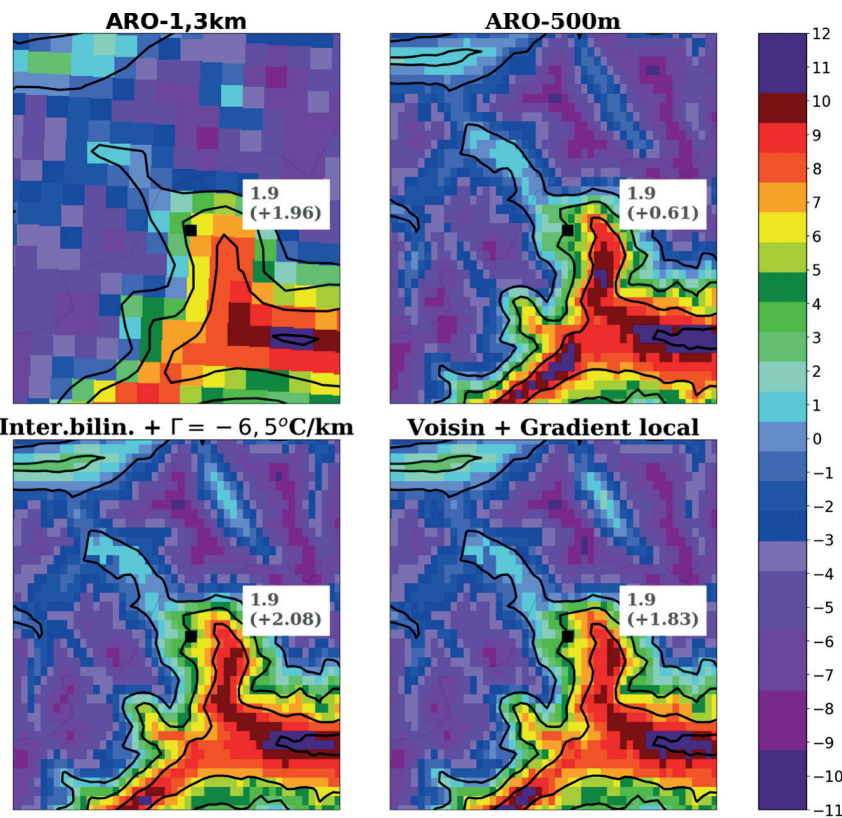

Figure 6. Champs de $T 2 m$ (en ${ }^{\circ} \mathrm{C}$ ) simulés par le modèle Arome $1,3 \mathrm{~km}$ (réseau de $0 \mathrm{~h}$ TU du 13 février 2020) et trois approches de descente d'échelle sur une portion des Alpes cottiennes et du val de Suse, à 15 heures d'échéance. L'observation à la station de Barcenisio (carré noir) est inscrite dans le rectangle blanc accompagnée du biais entre parenthèses. Les lignes de niveau sont tracées en noir. Le champ issu de la méthode « Voisin $+\Gamma=-6,5^{\circ} \mathrm{C} / \mathrm{km}$ », non représenté ici, est similaire aux champs obtenus avec les deux autres descentes d'échelle correctives. 
descentes d'échelle correctives se rapprochent de la simulation à haute résolution. À l'inverse des scores moyens qu'elle présente au-dessus de $1000 \mathrm{~m}$ (voir figure 4), cette dernière est ici la plus proche de l'observation (biais de $0,61{ }^{\circ} \mathrm{C}$ ) quand les autres méthodes présentent un biais au moins trois fois plus important (biais de $1,83{ }^{\circ} \mathrm{C}$ avec la méthode «Voisin $+\Gamma$ local » par exemple). Le profil de T2m correspondant à l'environnement de la station est affiché sur la figure 7. Si la régression linéaire calcule un gradient vertical pertinent de $-8,1{ }^{\circ} \mathrm{C} / \mathrm{km}$, en adéquation avec un profil sec de fœhn, on retrouve le biais décrit plus haut. Celui-ci est imputable à la variabilité horizontale de la température ou bien à l'erreur du modèle. En effet, alors que la différence d'altitude entre la station et le point (en bleu) le plus proche sur la grille $1,3 \mathrm{~km}$ n'est que de 13 mètres, $2^{\circ} \mathrm{C}$ séparent les valeurs associées.

\section{Inversion dans une vallée alpine}

Le 31 décembre 2019, un air calme sous des conditions anticycloniques a permis la formation d'une couche d'inversion dans les vallées savoyardes. La figure 8 illustre le champ de $T 2 m$ prévu à $6 \mathrm{~h}$ TU sur la combe de Savoie (cadre bleu sur la figure 4) par le modèle Arome 1,3 km, ainsi que par les différentes descentes d'échelle. L'inversion est bien représentée, en particulier par Arome $500 \mathrm{~m}$ où les températures descendent jusqu'à $-2{ }^{\circ} \mathrm{C}$ dans la vallée (« langue » bleue en bas à gauche de la figure associée). C'est d'ailleurs la simulation la plus proche de la température mesurée à la station d'Albertville-JO avec un biais

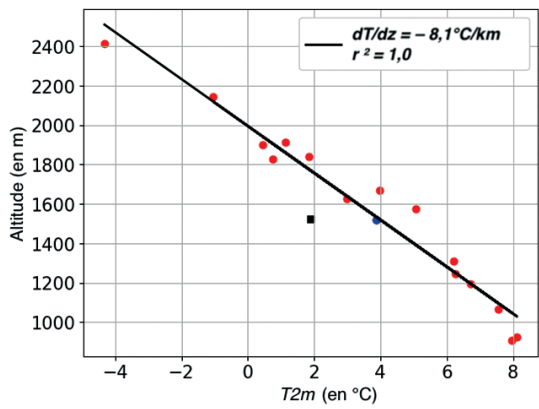

Figure 7. Profil vertical des données de $T 2 m$ (ronds rouges) associées aux 16 points du modèle Arome 1,3 km qui appartiennent à l'environnement de la station italienne Barcenisio (carré noir), le 13 février 2020 à 15 h TU. Le point du modèle le plus proche est en bleu. La droite calculée par la régression linéaire est tracée en noir. Le coefficient $r^{2}$, arrondi à 1,0 , traduit sa pertinence. de $1,7{ }^{\circ} \mathrm{C}$. Celui-ci est plus important pour les deux stations situées au nord, là où l'orographie est très resserrée. Les descentes d'échelle correctives se démarquent peu les unes des autres. L'interpolation bilinéaire renvoie un champ plus lisse, car elle assure davantage de continuité entre les données ; pour autant, elle ne donne pas les meilleurs résultats. Les simulations reposant sur une interpolation au point le plus proche sont très ressemblantes. Le gradient local calculé via la méthode de Sheridan et al. (2010) ne permet pas de restituer l'intensité de l'inversion telle qu'elle est observée et dont se rapproche Arome $500 \mathrm{~m}$. Deux raisons peuvent être invoquées pour expliquer cette contre-performance ; elles font l'objet de la prochaine section.

\section{Comment améliorer les descentes d'échelle correctives?}

Les descentes d'échelle correctives mises en œuvre ici reflètent une part des variabilités horizontale et verticale de la température. Grâce à son gradient calculé point par point, la méthode de Sheridan et al. (2010) est en outre capable de reproduire des stratifications thermiques particulières, éloignées de l'atmosphère standard et pourtant communes aux régions montagneuses. Or ses performances sont relativement faibles par rapport aux autres méthodes censées être moins précises. L'analyse faite sur l'inversion du 31 décembre 2019 permet de mieux
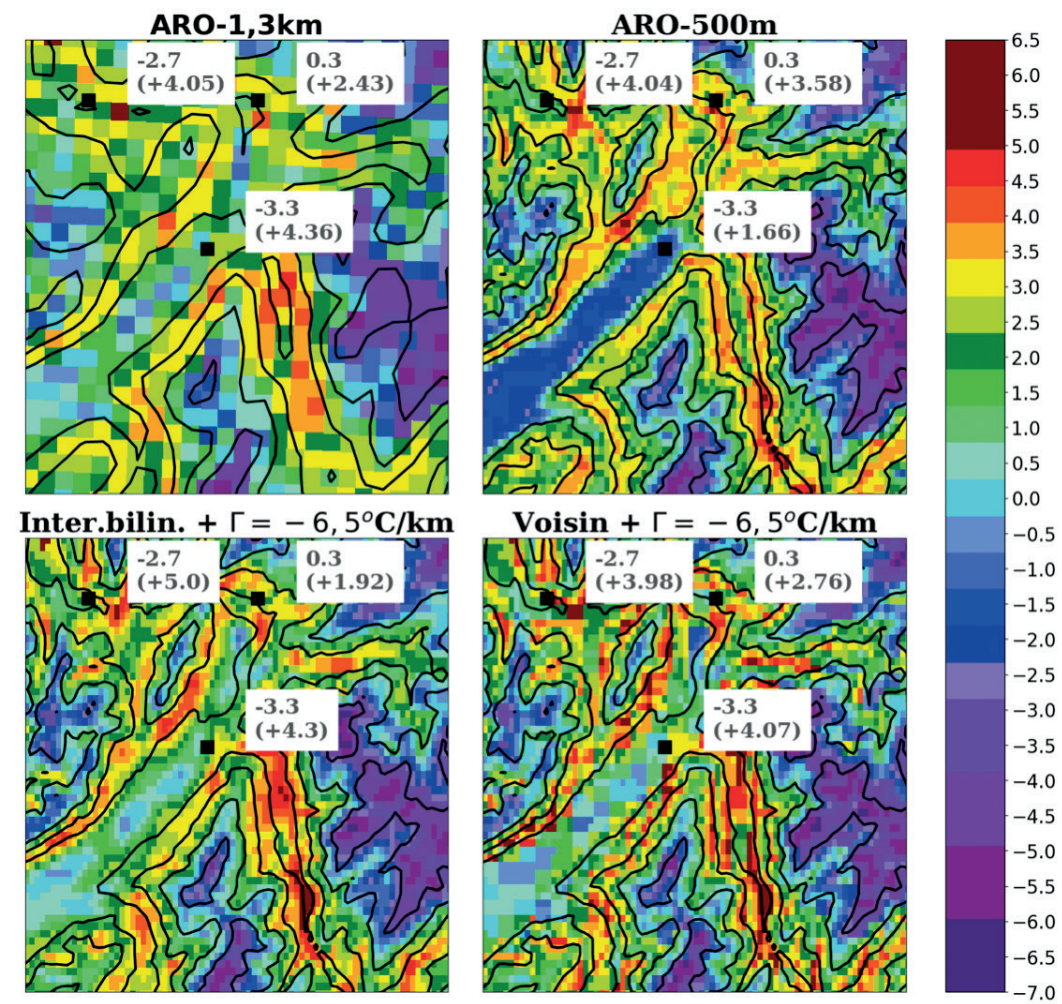

Voisin + Gradient local

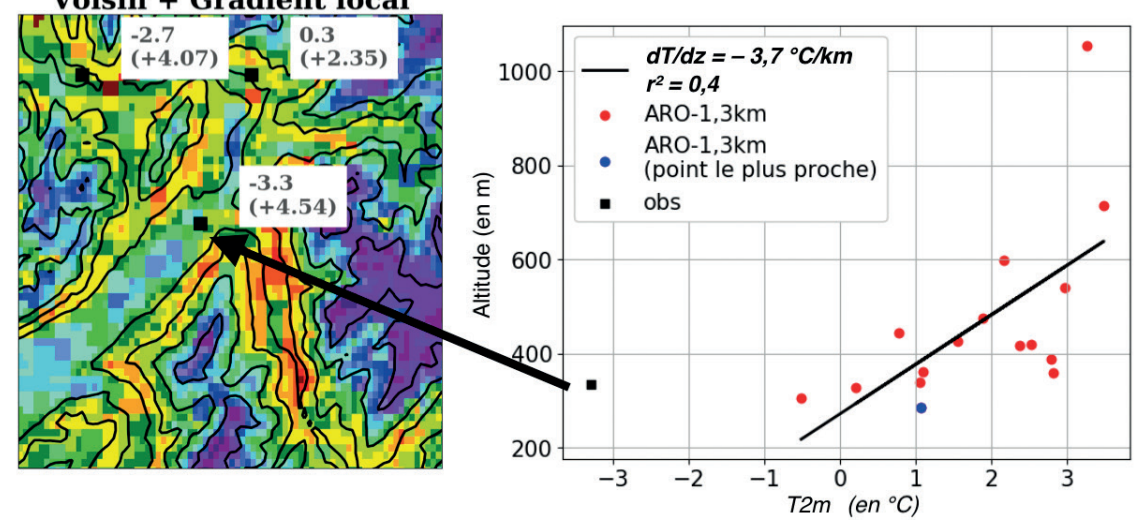

Figure 8. Champs de $T 2 \mathrm{~m}$ (en ${ }^{\circ} \mathrm{C}$ ) simulés par le modèle Arome $1,3 \mathrm{~km}$ (réseau de $0 \mathrm{~h}$ TU du 31 décembre 2019) et quatre approches de descente d'échelle sur la combe de Savoie, à 6 heures d'échéance. La flèche noire indique la station Albertville-JO dont un profil analogue à celui de la figure 7 est représenté en bas à droite. 
cerner ses limitations et leurs causes, afin d'identifier clairement les aspects sur lesquels une telle approche pourrait être enrichie.

\section{Ce qui peut être amélioré par une descente d'échelle corrective...}

Premièrement, l'hypothèse d'un gradient linéaire sur laquelle repose la régression est parfois inadaptée au profil de l'environnement considéré. Cet effet est en partie visible sur le graphique en bas à droite de la figure 8 . On a affiché les 16 points $T 2 m(z)$ du modèle (en rouge) appartenant à l'environnement de la station Albertville-JO dont l'observation est marquée d'un carré noir. $\mathrm{Si}$ on devine le profil de l'inversion à basse altitude, le point le plus élevé traduit plutôt une évolution nulle de la $T 2 m$, voire une décroissance, autrement dit le sommet de la couche d'inversion entre 800 et $1000 \mathrm{~m}$. La faible valeur du coefficient $r^{2}(0,4)$ indique une qualité médiocre de la régression linéaire qui coïncide avec une probable sous-estimation du gradient thermique $\left(+3,7^{\circ} \mathrm{C} / \mathrm{km}\right)$ à basse altitude. Dans ce cas, il conviendrait d'utiliser plus de points et/ou d'approcher le profil local par deux droites : l'une pour l'inversion proprement dite et l'autre pour l'atmosphère sus-jacente, les deux étant séparées par le « nez » de l'inversion. Cette suggestion fait partie des perspectives d'amélioration qui ne tiennent qu'à la méthodologie des descentes d'échelle. Outre une meilleure représentativité des profils, des facteurs environnementaux propres à chaque point de la grille fine peuvent être pris en compte comme le type de surface (prairies, forêts, neige, villes, plans d'eau...) ou l'exposition au rayonnement solaire, élément pertinent lorsque la maille est située sur la pente d'un relief. Ces caractéristiques sont déjà connues pour tous les points de la grille grossière et constituent autant de variables explicatives permettant d'élaborer un modèle correctif plus sophistiqué qu'une simple régression entre les données de température et d'altitude.

\section{... et ce qui ne le peut pas}

En observant le profil de la figure 8, on note que l'altitude au point du modèle Arome (en bleu) situé le plus près de la station Alberville-JO est presque identique à l'altitude de cette dernière. Or la prévision de $T 2 m$ y est d'environ $4{ }^{\circ} \mathrm{C}$ plus élevée. Aussi ce biais tient-il davantage à une erreur de modélisation qu'à une erreur d'interpolation. Plus

\section{Partie dynamique et partie physique d'un modèle. Quelles différences? Quels liens avec la résolution?}

Les variables pronostiques peuvent être décomposées en la somme d'une valeur moyenne, dont l'évolution est calculée par le schéma numérique du modèle (sa dynamique), et d'une fluctuation qui tient compte des processus sous-maille. C'est ce qui est fait pour la turbulence qui représente le mélange sous-maille dans le modèle. La fluctuation en elle-même peut être négligée, mais pas son influence sur l'évolution de la valeur moyenne (via un terme de "transport turbulent "), surtout au voisinage de la surface. Ce terme de transport turbulent n'est pas calculé dynamiquement mais approché par une paramétrisation qui relève de la physique du modèle. Dans Arome, la physique est codée pour tenir compte des processus turbulents

largement, les différences entre les simulations constatées sur la figure 5 sont faibles par rapport aux biais que le modèle Arome présente en zone de montagne. Ce dernier est trop chaud à basse altitude, entre $0 \mathrm{~h}$ et $9 \mathrm{~h}$ TU notamment. Le biais correspondant, de $0,5{ }^{\circ} \mathrm{C}$ en moyenne, est plus marqué au fond des vallées où les stations concernées renvoient les plus grands écarts (non montré). En altitude, au contraire, le modèle est trop froid de $1{ }^{\circ} \mathrm{C}$ l'après-midi et de $3{ }^{\circ} \mathrm{C}$ la nuit et le matin. Ces biais, déjà identifiés par des études antérieures (Paci et al., 2016 ; Vionnet et al., 2016 ; Dombrowski-Etchevers et al., 2017 ; Gouttevin et al., 2018), sont régulièrement signalés par les prévisionnistes régionaux, notamment en situation d'isothermie lorsqu'ils impactent la prévision de la limite pluie-neige. Les hypothèses émises pour les justifier impliquent souvent des paramétrisations trop frustes dans la physique du modèle, en particulier les schémas de turbulence ou de manteau neigeux. Les erreurs intrinsèques au modèle ne peuvent pas être traitées directement par ces méthodes de descente d'échelle, au risque d'introduire un débiaisage nuisible à leur interprétation et à leur robustesse. Enfin, force est de constater de taille inférieure au kilomètre. En poussant la résolution du modèle à 500 mètres, la dynamique et la physique d'Arome se recouvrent déjà partiellement : on parle de "zone grise " de la turbulence. Cette problématique devient saillante dans la modélisation de certains processus hectométriques. Son rôle dans l'amplification des biais observés avec Arome $500 \mathrm{~m}$ est une supposition qu'il convient de moduler en fonction des défauts relatifs à la physique pure du modèle. Parmi eux, l'hypothèse selon laquelle le "transport turbulent " s'effectue dans la direction verticale uniquement et non dans les deux autres directions horizontales : une hypothèse contestable en terrain complexe, qui plus est à 500 mètres de résolution.

que certains défauts du modèle Arome se retrouvent dans sa version haute résolution à $500 \mathrm{~m}$. À l'image du biais froid d'altitude, ils sont parfois exacerbés pour des raisons qui n'ont pas encore été étudiées. Une physique en partie inadaptée vis-à-vis de la dynamique à $500 \mathrm{~m}$ constitue cependant une piste de réflexion (voir encadré 2 ). Peu importe la stratégie adoptée, descente d'échelle corrective ou non, investiguer la physique du modèle apparaît donc comme une étape nécessaire dans la course à la résolution hectométrique !

\section{Conclusions}

La prévision du risque d'avalanche, entre autres, repose sur un suivi précis du manteau neigeux dont l'évolution dépend généralement de processus actifs à des échelles sous-maille, y compris pour le modèle Arome à $1,3 \mathrm{~km}$ de résolution. Afin d'augmenter la résolution à faible coût numérique, des descentes d'échelle correctives ont donc été développées ; elles sont ici évaluées pour la température à 2 mètres sur les Alpes occidentales. Une interpolation horizontale reflète la variabilité spatiale de

\section{Quelles approches de descente d'échelle pour les autres variables?}

En montagne, la température est loin d'être le seul paramètre à enjeux. Des descentes d'échelle correctives existent pour le vent, les précipitations, le rayonnement et l'humidité relative. Cette dernière a d'ailleurs fait l'objet d'une étude parallèle au travail présenté dans cet article. L'évolution de l'humidité avec l'altitude est plus complexe que pour la température. Une fois convertie en température du point de rosée $(T d)$, l'approximation linéaire devient une hypothèse pertinente.

Deux corrections de hauteur, analogues à celles qui viennent d'être présentées, sont ainsi suggérées : la première est fondée sur un gradient climatologique de $T d$ fixé à sa moyenne annuelle de $-5,5{ }^{\circ} \mathrm{C} / \mathrm{km}$ (Liston et Elder, 2006 ; Kunkel, 1989), la seconde sur un gradient local calculé suivant la méthode de Sheridan et al. (2010). Non illustrée ici, leur évaluation n'a toutefois pas montré d'amélioration par rapport à une descente d'échelle dépourvue de correction verticale. 
la température à l'échelle kilométrique. Elle est accompagnée d'une correction d'altitude qui traduit la différence de hauteur entre le relief de la grille ciblée (à $500 \mathrm{~m}$ de résolution) et celui du modèle. Plusieurs approches testées montrent des performances similaires à celles du modèle Arome 500 m, mais leur biais par rapport aux observations reste marqué, notamment en altitude et au fond des vallées. En outre, le biais froid d'Arome $500 \mathrm{~m}$ augmente avec l'altitude. Ces deux constatations mettent en avant deux axes de recherche : (1) affiner les corrections en fonction de certains effets locaux et du profil thermique de l'atmosphère modélisée, (2) adapter la physique du modèle. Analyser l'impact des descentes d'échelle correctives sur d'autres variables atmosphériques constitue finalement une prochaine étape (voir encadré 3).

\section{Remerciements}

La rédaction de cet article s'inscrit dans la continuité d'un projet de fin d'étude réalisé en troisième année de l'École nationale de la météorologie (ENM). Nos remerciements vont aux membres du CNRM, Ryad El Khatib, Pascal Marquet, Jean-Marcel Piriou et Éric Bazile, ainsi que Véronique Mathiot et Francis Pouponneau pour la direction des opérations de MétéoFrance. Leur aide, tant technique que scientifique, a été précieuse. Enfin, nous tenons à souligner l'apport bénéfique des retours d'expérience fournis par Joël Marceaux et les prévisionnistes de la Direction CentreEst.

\section{Bibliographie}

Dombrowski-Etchevers I., Vionnet V., Quéno L., 2017. Pertinence des prévisions météorologiques à l'échelle kilométrique pour la modélisation du manteau neigeux en montagne. La Météorologie, 99, 25-34.

Gouttevin I., Vionnet V., Karbou F., Merzisen H., Deliot. Y., 2018. To the origin of the temperature bias in the arome numerical weather model: investigations at a high altitude site. Proceedings of the International Snow Science Workshop, Innbruck, Austria, 756-758.

Kunkel K.E., 1989. Simple procedures for extrapolation of humidity variables in the mountainous Western United States. J. Clim., 2, 656-669.

Liston G., Elder K., 2006. A meteorological distribution system for high resolution terrestrial modeling (micromet). J. Hydrometeorol., 7, $217-234$.

Paci A., Staquet C., Allard J. et al., 2016. The Passy-2015 field experiment: atmospheric dynamics and air quality in the Arve River Valley. Pollut. Atmos., $231-232$.

Sheridan P., Smith S., Brown A., Vosper S., 2010. A simple height-based correction for temperature downscaling in complex terrain. Meteorol. Appl., 17, 329-339.

Soci C., Landelius T., Bazile E., Undén P., Mahfouf J.-F., Martin E., Besson F., 2011. D 2.10 Comparison of existing ERAMESAN with SAFRAN downscaling. EURO4M Project, Report.

Vionnet V., Dombrowski-Etchevers I., Lafayses M, Quéno L., Seity Y., Bazile E., 2016. Numerical weather forecasts at kilometer scale in the French Alps: Evaluation and application for snowpack modeling. J. Hydrometeorol., 17, 2591-2614. 